\title{
THE CONTINUITY OF ARENS' PRODUCT ON THE STONE-ČECH COMPACTIFICATION OF SEMIGROUPS
}

\author{
BY
}

\author{
NICHOLAS MACRI( $\left.{ }^{1}\right)$
}

ABSTRACT. A discrete semigroup is said to have the compact semigroup property (c.s.p.) [the compact semi-semigroup property (c.s.s.p.)] if the multiplication Arens' product, on its Stone-Čech compactification, is jointly [separately] $w^{*}$-continuous. We obtain an algebraic characterization of those semigroups which have c.s.p. by characterizing algebraically their almost periodic subsets. We show that a semigroup has c.s.p. if and only if each of its subsets is almost periodic. This characterization is employed to prove that for a cancellation semigroup to have c.s.p., it is necessary and sufficient that each of its countable subsets be almost periodic. We answer in the negative a heretofore open question-is c.s.p. equivalent to c.s.s.p.

1. Introduction. It is well known [4] that a discrete semigroup $S$ is imbedded homeomorphically and densely in its Stone-Čech compactification $\beta(S)$. A multiplication called Arens' product [1], denoted $\odot$, can be placed on $\beta(S)$. With this multiplication, $\beta(S)$ becomes a compact set and a semigroup which contains an isomorphic copy of $S$. However, $\beta(S)$ equipped with $\odot$ may fail to be a compact semigroup in that $\odot$ need not be jointly continuous on $\beta(S)$, as some examples in $\$ 3$ point out. In fact, $\odot$ may even fail to be separately continuous on $\beta(S)$. An example of a semigroup for which this occurs appears in $\$ 5$. In this paper we are concerned with the questions of determining which semigroups $S$ have separate or joint continuity of $\odot$ on $\beta(S)$.

In $\$ 2$, basic definitions and a discussion of the methods and terminology we will use are given. We will say that a discrete semigroup $S$ has the compact semigroup property, c.s.p., (compact semi-semigroup property, c.s.s.p.) if $\odot$ is jointly (separately) continuous on $\beta(S)$.

In $\$ 3$ we give an algebraic characterization of those semigroups $S$ which

Presented to the Society, January 17, 1972; received by the editors January 6, 1972 and, in revised form, July 23, 1973.

AMS (MOS) subject classifications (1970). Primary 43A60; Secondary 22A15, $22 \mathrm{~A} 20$.

Key words and phrases. Arens' product, joint continuity, separate continuity, StoneČech compactification, almost periodic, weakly almost periodic.

(1) This paper is a portion of the author's Temple University thesis prepared under the direction of Professor Theodore Mitchell. 
have c.s.p. First, we characterize algebracially the almost periodic subsets of $S$. Then we show that a semigroup $S$ has c.s.p. if and only if every one of its subsets is almost periodic. We apply this characterization to various semigroups to determine whether or not they have c.s.p. As a final result in this section, we prove that for a cancellation semigroup to have c.s.p., it is necessary and sufficient that each of its countable subsets be almost periodic.

In $\$ 5$ where we discuss semigroups with c.s.s.p. (i.e., $\odot$ is separately continuous on $\beta(S)$ ) we answer, in the negative, a heretofore open question-is c.s.p. equivalent to c.s.s.p.-by constructing a semigroup with c.s.s.p. but not c.s.p.

2. Preliminaries. A topological semigroup is a semigroup with a Hausdorff topology such that the multiplication is jointly continuous. Let $S$ be a discrete semigroup. We denote by $m(S)$ the space of all bounded real valued functions on S. $m(S)$ is a Banach space with the supremum norm.

Let $S$ be a semigroup (not necessarily having an identity). For $f$ in $m(S)$ and $x \in S$, the left (right) translates of $f$ by $x$ are defined by $f_{x}(y)=f(x y)\left(f^{x}(y)=\right.$ $f(y x))$. Clearly $f_{x}, f^{x} \in m(S)$ for all $x \in S$. Let $m(S) *$ denote the space of bounded real linear functionals on $m(S)$.

Define a map $\rho: S \rightarrow m(S)^{*}$ by $s \rightarrow \delta_{s}$, where $\delta_{s}(f)=f(s)$ for all $f \in m(S)$. The $w^{*}$-closure of $\rho(S)$ in $m(S)^{*}$, denoted by $\beta(S)$, is called the Stone-Čech compactification of $S$. A well-known multiplication [1] called Aren's product, denoted by $\odot$, can be defined on $m(S)^{*}$ in such a way that $m(S)^{*}, \odot$ is a semigroup. Let $\mu, \lambda \in m(S)^{*}$. Define,

$$
(\mu \odot \lambda)(f)=\mu(b) \text { for all } f \in m(S),
$$

where $b(x)=\lambda\left(f_{x}\right)$ for all $X \in S$. Then $m(S)^{*}$ is a semigroup under $\odot$, and $\beta(S)$ is a subsemigroup and a compact set which contains a dense isomorphic and homeomorphic copy, $\rho(S)$, of $S$.

The only topology on the semigroup $S$ that we consider in this paper will be the discrete topology, and $\beta(S)$ will al ways carry the $w^{*}$-topology of $m(S)^{*}$.

Definition. A semigroup $S$ is said to have the compact semigroup property (c.s.p.) if $\odot$ is jointly continuous on $\beta(S)$.

Definition. A semigroup $S$ is said to have the compact semi-semigroup property (c.s.s.p.) if $\odot$ is separately continuous on $\beta(S)$.

Definition. $f \in m(S)$ is called weakly almost periodic if $\left\{f^{x} \mid x \in S\right\}$ is relatively weakly compact.

Definition. $f \in m(S)$ is said to be almost periodic if $\left\{f^{x} \mid x \in S\right\}$ is relatively compact in the norm topology.

For $A \subset S$ we will denote by $C_{A}$ the characteristic function of $A$. We let

$$
A x^{-1}=\{t \mid t \in S \text { and } t x \in A\} \text {. }
$$


Note that, for $H \subset S$ and $x \in S, C_{H}^{x}=C_{H x-1}$. The set of almost periodic functions on $S$ will be denoted by $A(S)$. The set of weakly almost periodic functions on $S$ will be denoted by $W(S)$. It is well known (see Pym [8, p. 93]) that $A(S)$ and $W(S)$ are norm closed subspaces of $m(S)$. The product $\odot$, previously defined, is a special case of what J. Pym [7] calls evolution on $m(S) *$. We will make use of Lemma 6.1 and Theorems 5.2 and 5.4 in Pym's work [8].

Remark. Part (iii) of Lemma 6.1 holds if the word "convolution" is replaced by the word "evolution." Theorem 6.2 is still valid if the phrase "domain for a convolution in $F^{*}$ " is replaced by the phrase "domain for an evolution in $F^{*}$."

The following theorem is a restatement of a portion of [8, Theorem 4.3].

Theorem 1. Let $S$ be a semigroup, and $F$ a Banacb subspace of $m(S)$ sucb that $F$ is a domain for convolution in $F^{*}$. Then the following statements are equivalent:

(i) $f^{T} \in F$ for all $f \in F$ and $T \in F^{*}$, and convolution is identical with evolution in $F^{*}$.

(ii) Each $f$ in $F$ is weakly almost periodic.

The following result is an immediate, but apparently previously unrecognized, special case of Theorems 6.2 and 4.3 of Pym [8].

Theorem 2. Let $S$ be a semigroup. Then $S$ bas c.s.s.p. if and only if convolution is identical with evolution on $m(S) *$.

Proof. Let $F=m(S)$, and apply Theorem 6.2(ii) of Pym [8] and the above restatement of Theorem 4.3 of Pym [8].

3. The characterization of semigroups with the compact semigroup property.

Definition 1. We will call a subset $H$ of a semigroup $S$ almost periodic if $C_{H} \in A(S)$.

Theorem 1. If $H \subset S$, where $S$ is a semigroup, then $H$ is almost periodic if and only if $\left\{H x^{-1} \mid x \in S\right\}$ is finite.

Proof. Since, for $H \subset S$ and $x \in S, C_{H}^{x}=C_{H x-1}$ we have that $H$ is almost periodic if and only if $\left\{C_{H_{x}-1} \mid x \in S\right\}$ is finite.

Theorem 2. Let $S$ be a semigroup. Then $m(S)=A(S)$ if and only if every $H \in 2^{S}$ is almost periodic.

Proof. The theorem is a consequence of the facts that $m(S)$ is the norm closure of the space spanned by the characteristic functions of the subsets of $S$ and that $A(S)$ is norm closed in $m(S)$.

Corollary 1. A semigroup $S$ has c.s.p. ( $\odot$ is jointly $w^{*}$-continuous on $\beta(S)$ ) if and only if, for each $H \in 2^{S},\left\{H x^{-1} \mid x \in S\right\}$ is finite. 
Proof. If, for each $H \in 2^{S},\left\{H x^{-1} \mid x \in S\right\}$ is finite then by Theorems 1 and 2 above we have that $m(S)=A(S)$. By Theorem 5.4 of Pym [8], we have, since $\odot$ is evolution on $m(S)^{*}$, that $\odot$ is jointly $w^{*}$-continuous on $\beta(S)$. So $S$ has c.s.p.

If $S$ has c.s.p. then, by the remark made in $\$ 2$ and Theorem 6.2 of Pym [8], we have that $m(S)=A(S)$ and the corollary is proved.

The following examples with the exception of number 9 occur in the work of H. Mankowitz [6]. Completely different and simple proofs can be easily obtained using our characterization. We include only the proof of number 6 .

Examples. 1. Let $S$ be an arbitrary nonempty set, and define a product by $a b=b$ for any $a, b \in S$. Then $S$ is a semigroup with c.s.p.

2. Let $S$ be an arbitrary nonempty set, and define a product by $a b=a$ for any $a, b \in S$. Then $S$ is a semigroup with c.s.p.

3. Let $S$ be the semigroup of positive integers with $a b=\max (a, b)$. Then $S$ does not have c.s.p.

4. Let $S$ be the semigroup of positive integers with $a b=\min (a, b) . S$ does not have c.s.p.

5. Let $S$ be any nonempty set and $x_{0}$ a fixed element of $S$. Define $a b=x_{0}$. Then the semigroup $S$ has c.s.p.

6. The semigroup of positive integers $N$ under addition does not have c.s.p.

Proof. Let $H=\left\{2^{k} \mid k=1,2, \ldots\right\}$. Let $2^{n_{1}}, 2^{n_{2}} \in H$ with $n_{1}<n_{2}$. Then

$$
2^{n}{ }^{1} \in H\left(2^{n}\right)^{-1} \text { and } 2^{n_{1}} \notin H\left(2^{n}\right)^{-1}
$$

So $\left\{H a^{-1} \mid a \in H\right\}$ is infinite and since $\left\{H x^{-1} \mid x \in N\right\} \supsetneq\left\{H a^{-1} \mid a \in H\right\}$ we have by Corollary 1 that $N$ does not have c.s.p.

7. The set of positive real numbers under addition does not have c.s.p.

8. The group of integers under addition does not have c.s.p.

9. The semigroup of positive integers $Z$ under multiplication does not have c.s.p.

10. Any finite semigroup has c.s.p.

Remark. Let $(S, \cdot)$ be a semigroup. For $a, b \in S$ set $a \times b=b$. $a$. Then $(S, x)$ is a semigroup and if $(S,$.$) has c.s.p. (c.s.s.p.) then (S, X)$ has c.s.p. (c.s.s.p.). This follows from the well-known fact that equivalent definitions of almost periodic and weakly almost periodic functions can be formed using left translates in place of right translates (see page 167 of [5] and Proposition 7 of [3]) and from Theorem 5.4. of [8] since $m(S)=A(S)(m(S)=W(S))$.

\section{Applications of the characterization.}

Theorem 1. A semigroup $S$, sucb that $S S=\{a b \mid a, b \in S\}$ is finite, need not bave c.s.p. 
Proof. Let $S$ be any infinite set. Let 0 and 1 be two distinguished elements of $S$. Define

$$
\begin{aligned}
a b & =1 \text { for } a=b, \\
& =0 \text { for } a \neq b,
\end{aligned} \text { and } a, b \neq 0,1,
$$

and $0 a=a 0=1 a=a 1=0$ for all $a \in S$.

$S$ is clearly a semigroup and since $\left\{\{1\} a^{-1} \mid a \in S\right\}$ is infinite we have that $S$ does not have c.s.p.

If additional conditions are imposed on $S$, then it will perhaps have c.s.p. If for instance we had that for each $A \subset S S$ there exists an $a \in S$ such that $A a \subset A$ and $(S S-A) a \subset S S-A, S$ would have c.s.p.

Theorem 2. Let $S$ be a cancellation semigroup with the property that every countable subset is almost periodic. Then $S$ bas c.s.p.

Proof. Suppose $H \subset S$ is such that $\left\{H x^{-1} \mid x \in S\right\}$ is infinite. Define, for $(x, y) \in S \times S, x \sim y$ iff $H x^{-1}=H y^{-1}$. $\sim$ is clearly an equivalence relation and since $\left\{H x^{-1} \mid x \in S\right\}$ is infinite, we have an infinite number of equivalence classes. Next form the set $X$ by selecting one element from each equivalence class omitting that class, $C_{s}$, if it exists, for which $H s^{-1}=\varnothing$. So $X$ is infinite, and, for $x \in \mathrm{X}, H x^{-1} \neq \varnothing$.

1. We may assume that for $t \in H$ there exists $x \in X$ such that $\{t\} x^{-1} \neq \varnothing$.

2. For $t \in H$ we have that $\left\{\{t\} x^{-1} \mid x \in X\right\}$ is finite, say expressed as $\left\{\{t\} x_{1}^{-1},\{t\} x_{2}^{-1}, \ldots,\{t\} x_{n}^{-1}\right\}$. This is so since every countable subset of $S$ is assumed to be almost periodic.

Notice that, for $t \in H,\{t\} x^{-1}$ has at most one point since if $u$ and $v \in\{t\} x^{-1}$ then $u x=t=v x \rightarrow u=v$.

3. For $x \in \mathrm{X}-\left\{x_{1}, \ldots, x_{n_{t}}\right\},\{t\} x^{-1}=\varnothing$; otherwise $\{t\} x^{-1}=\{t\} x_{k}^{-1} \neq \varnothing$ for some $1 \leq k \leq n_{t}$ and so for some $s \in S$ we have $s x=t=s x_{k} \rightarrow x=x_{k}$, a contradiction.

Let $t_{1} \in H$ and $\left\{x_{11}, x_{12}, \ldots, x_{1 n_{1}}\right\}$ be that subset of $\mathbf{X}$ such that $\left\{t_{1}\right\} x_{1 i}^{-1} \neq \varnothing, 1 \leq i \leq n_{1}$. Now then there exists $t_{2} \in H-\left\{t_{1}\right\}$ such that, for some $x_{21} \in X-\left\{x_{11}, \ldots, x_{1 n_{1}}\right\},\left\{t_{2}\right\} x_{21}^{-1} \neq \varnothing$ and $\left\{t_{2}\right\} x_{21}^{-1} \in S-\left\{\left\{t_{1}\right\} x_{11}^{-1}, \ldots,\left\{t_{1}\right\} x_{1 n_{1}}^{-1}\right\}$. This holds since otherwise $H x^{-1} \subset\left\{\left\{t_{1}\right\} x_{11}^{-1}, \ldots,\left\{t_{1}\right\} x_{1 n_{1}}^{-1}\right\}$ for every $x \in X$.

But by $2,\left\{\left\{t_{1}\right\} x_{11}^{-1}, \ldots,\left\{t_{1}\right\} x_{1 n_{1}}^{-1}\right\}$ is finite so that $\left\{H x^{-1} \mid x \in X\right\}$ is finitea contradiction. Let $\left\{x_{21}, x_{22}, \ldots, x_{2 n_{2}}\right\}$ be the set of those $x_{2 j} \in \mathbf{X}$ such that $\left\{t_{2}\right\} x_{2 j}^{-1} \neq \varnothing$. Again, there exists $t_{3} \in H-\left\{t_{1}, t_{2}\right\}$ such that, for some $x_{31} \in \mathbf{X}-$ $\left\{x_{11}, x_{12}, \ldots, x_{1 n_{1}}, x_{21}, \ldots, \dot{x}_{2 n_{2}}\right\},\left\{t_{3}\right\} x_{31}^{-1} \neq \varnothing$ and

$$
\left\{t_{3}\right\} x_{31}^{-1} \in S-\left\{\left\{t_{1}\right\} x_{11}^{-1}, \ldots,\left\{t_{1}\right\} x_{1 n_{1}}^{-1},\left\{t_{2}\right\} x_{21}^{-1}, \ldots,\left\{t_{2}\right\} x_{2 n_{2}}^{-1}\right\}
$$


since otherwise $H x^{-1}$ would be a subset of $\left\{\left\{t_{1}\right\} x_{11}^{-1}, \ldots,\left\{t_{1}\right\} x_{1 n_{1}}^{-1},\left\{t_{2}\right\} x_{21}^{-1}\right.$, $\left.\ldots,\left\{t_{2}\right\} x_{2 n_{2}}^{-1}\right\}$ for every $x \in X$. But as before, by $2,\left\{\left\{t_{1}\right\} x_{11}^{-1}, \ldots,\left\{t_{1}\right\} x_{1 n_{1}}^{-1}\right.$, $\left.\left\{t_{2}\right\} x_{21}^{-1}, \ldots,\left\{t_{2}\right\} x_{2 n_{2}}^{-1}\right\}$ is finite and so $\left\{H x^{-1} \mid x \in X\right\}$ is finite-a contradiction. In general then, for any $k$, there exists $t_{k} \in H-\left\{t_{1}, \ldots, t_{k-1}\right\}$ such that, for some $x_{k 1} \in \mathrm{X}-\left\{x_{11}, \ldots, x_{1 n_{1}}, x_{21}, \ldots, x_{2 n_{2}}, \ldots, x_{k-1,1}, \ldots, x_{k-1, n_{k-1}}\right\}$, $\left\{t_{k}\right\} x_{k 1}^{-1} \neq \varnothing$ and

$$
\begin{array}{r}
\left\{t_{k}\right\} x_{k 1}^{-1} \in S-\left\{t_{1}\right\} x_{11}^{-1}, \ldots,\left\{t_{1}\right\} x_{1 n_{1}}^{-1},\left\{t_{2}\right\} x_{21}^{-1}, \ldots,\left\{t_{2}\right\} x_{2 n_{2}}^{-1}, \ldots, \\
\left.\left\{t_{k-1}\right\} x_{k-1,1}^{-1}, \cdots,\left\{t_{k-1}\right\} x_{k-1, n_{k-1}}^{-1}\right\} .
\end{array}
$$

For any $k$ define $\left\{x_{k 1}, \ldots, x_{k n_{k}}\right\}$ to be that subset of $X$ of minimal finite cardinality such that $\left\{t_{k}\right\}\left\{x_{k 1}, \cdots, x_{k n_{k}}\right\}^{-1}=\left\{t_{k}\right\} X^{-1}$.

4. Now form $T_{n}=\left\{t_{1}, \ldots, t_{n}\right\} ; X_{n}=\left\{x_{11}, x_{21}, \ldots, x_{n 1}\right\}$. Let

$$
T=\bigcup_{n=1}^{\infty} T_{n}, \quad X^{\prime}=\bigcup_{n=1}^{\infty} X_{n}
$$

Now notice the following:

If, for $t_{k} \in T$ and $x \in X^{\prime},\left\{t_{k}\right\} x^{-1} \neq \varnothing$ then $\left\{t_{k}\right\} x^{-1} \in\left\{\left\{t_{k}\right\} x_{k 1}^{-1}, \ldots,\left\{t_{k}\right\} x_{k n_{k}}^{-1}\right\}$ and

$$
\left\{t_{k}\right\} x^{-1} \notin\left\{t_{1}, \cdots, t_{k-1}\right\} X^{-1} \text {. }
$$

5. This implies that $\left\{T x_{i 1}^{-1} \mid x_{i 1} \in \mathbf{X}^{\prime}\right.$ (i.e. for $\left.\left.i=1,2, \ldots\right)\right\}$ is infinite. As a matter of fact no two of the elements in this set are the same for $i \neq j$. Consider $T x_{i 1}^{-1}$ and $T x_{j 1}^{-1}$, with $i \neq j$. Then $\left\{t_{i}\right\} x_{i 1}^{-1} \neq \varnothing$ and $\left\{t_{j}\right\} x_{j 1}^{-1} \neq \varnothing$. Also $T x_{i 1}^{-1}=$ $\left\{\left\{t_{i}\right\} x_{i 1}^{-1},\left\{t_{i+1}\right\} x_{i 1}^{-1}, \ldots\right\}, T x_{j 1}^{-1}=\left\{\left\{t_{j}\right\} x_{j 1}^{-1},\left\{t_{j+1}\right\} x_{j 1}^{-1}, \ldots\right\}$. Suppose $\left\{t_{i+\mu}\right\} x_{i 1}^{-1}=$ $\left\{t_{j+\nu}\left\{x_{j 1}^{-1} \neq \varnothing\right.\right.$, for some integers $\mu, \nu \geq 0$. Then note that $i+\mu \neq j+\nu$. For otherwise $t_{i+\mu}=t_{j+\nu}$ and then $\left\{t_{i+\mu}\right\} x_{i 1}^{-1} x_{i 1}=t_{i+\mu}=t_{j+\nu}=\left\{t_{j+\nu}\right\} x_{j 1}^{-1} x_{j 1} \rightarrow x_{i 1}=$ $\boldsymbol{x}_{j 1}$, a contradiction. Since $i+\mu \neq j+\nu$, it follows from $(*)$, with $k$ the larger of $i+\mu, j+\nu$, that $\left\{t_{i+\mu}\right\} x_{i 1}^{-1} \neq\left\{t_{j+\nu}\left\{x_{j 1}^{-1}\right.\right.$. Therefore $T x_{i 1}^{-1} \neq T x_{j 1}^{-1}$ for $i \neq j, i, j=$ $1,2, \ldots$.

6. Therefore $T$ is not almost periodic, which is a contradiction since $T$ is countable. Hence, for $H \subset S,\left\{H x^{-1} \mid x \in S\right\}$ is finite. Therefore $S$ has c.s.p.

As some other applications of the characterization one can easily obtain some already known results [6] such as:

1. Subsemigroups of semigroups with c.s.p. have c.s.p.

2. Homomorphic images of semigroups with c.s.p. have c.s.p.

We can easily get that the cartesian product of a semigroup with c.s.p. and a finite semigroup has c.s.p. But if the condition of finiteness for one of the semigroups is dropped the product may fail to have c.s.p. 
5. Separate continuity of $\odot$ on $\beta(S)$. In this chapter we will make use of the following theorem due to Grothendieck [3].

Theorem 1. Let $E$ be any topological space and let $C(E)$ denote the Banach space of all bounded continuous functions on $E$. Then a set $A \subseteq C(E)$ is relatively weakly compact if and only if it is bounded and it is impossible to choose sequences $\left\{f_{i}\right\}$ in $A$ and $\left\{x_{j}\right\}$ in $E$ such that the limits $\operatorname{Lim}_{i} \operatorname{Lim}_{j} f_{i}\left(x_{j}\right)$ and $\operatorname{Lim}_{j} \operatorname{Lim}_{i} f_{i}\left(x_{j}\right)$ both exist and are distinct.

Let $S$ be a semigroup and $f \epsilon m(S)$. Grothendieck points out that $f$ is weakly almost periodic if and only if $\operatorname{Lim}_{i} \operatorname{Lim}_{j} f\left(x_{i} y_{j}\right)$ and $\operatorname{Lim}_{j} \operatorname{Lim}_{i} f\left(x_{i} y_{j}\right)$ are equal whenever they exist. $\left(\left\{x_{i}\right\},\left\{y_{j}\right\}\right.$ are sequences in $\left.S_{\text {. }}\right)$

Theorem 2. For a semigroup $S, m(S)=W(S)$ if and only if $C_{H}$ is weakly almost periodic for every $H \subset S$.

Proof. Since $W(S)$ is a Banach space and $m(S)$ is the norm closure of the linear span of the characteristic functions of subsets of $S$, the theorem follows.

Remark. In dealing with the question of whether $m(S)=W(S)$ implies that $m(S)=A(S)$, we need only consider the characteristic functions of the subsets of $S$.

Theorem 3. There exists a semigroup $S$ such that $m(S)=W(S)$ but $m(S) \neq$ $A(S)$.

Proof. Let $S$ be the semigroup in the proof of Theorem $1, \$ 4$. Consider now $C_{\{t\}}^{a_{i}}\left(y_{j}\right)$ where $\left\{a_{i}\right\},\left\{y_{j}\right\}$ are sequences in $S$ and $t=0$ or $t=1$. We consider two cases.

A. Neither sequence $\left\{a_{i}\right\},\left\{y_{j}\right\}$ has an infinite number of 0 's or 1's.

B. One of the sequences (possibly both) has an infinite number of 0 's or 1's.

Suppose A: We consider two subcases $t=1$ and $t=0$. Suppose $t=1$ and that

$$
\operatorname{Lim}_{i} \operatorname{Lim}_{j} C_{\{1\}}^{a_{i}}\left(y_{j}\right)=\operatorname{Lim}_{i} \operatorname{Lim}_{j} C_{\{1\}}\left(y_{j} a_{i}\right)=1
$$

Then there exists $N$ such that for each $i \geq N$ there exists $M(i)$ such that for $j \geq M(i)$

$$
C_{\{1\}}\left(y_{j} a_{i}\right)=1 \text { for } j \geq M(i) .
$$

This means that $y_{j}=a_{i}$ for $j \geq M(i)$. So taking $i=N$ and $M=M(N)$ we have that $y_{M}=y_{M+1}=y_{M+2}=\cdots=a_{N}$. But then on taking $i=N+P$ and applying the above we have that $a_{N+P}=a_{N}$. So $a_{N}=a_{N+1}=a_{N+2}=\cdots$.

But this implies that $\operatorname{Lim}_{j} \operatorname{Lim}_{i} C_{\{1\}}^{a_{i}}\left(y_{j}\right)=\operatorname{Lim}_{j} \operatorname{Lim}_{i} G_{1}\left(y_{j} a_{i}\right)=1$, So then if $\operatorname{Lim}_{j} \operatorname{Lim}_{j} C_{\{1}^{a_{i}}\left(y_{j}\right)=1, \operatorname{Lim}_{j} \operatorname{Lim}_{i} C_{\{1\}}^{a_{i}^{i}}\left(y_{j}\right)$ exists and is also 1 . A similar argument will give 
that if $\operatorname{Lim}_{j} \operatorname{Lim}_{i} C_{\{1\}}^{a_{i}}\left(y_{j}\right)=1$ then $\operatorname{Lim}_{i} \operatorname{Lim}_{j} C_{1\}}^{a_{i}}\left(y_{j}\right)$ exists and is also 1 .

We have in this subcase that if one of the iterated limits exists and is 1 then the other must exist and be equal to 1 .

Suppose now that one of the iterated limits exists and is 0 . Then if the other iterated limit exists, it too must be 0 , since if it were 1 , the first iterated limit would also have to equal 1 , by what was proved above. In summary then we have that, for $t=1$, if the iterated limits both exist, they must be equal.

Next suppose $t=0$ and that

$$
\operatorname{Lim}_{i} \operatorname{Lim}_{j} C_{\{0\}}^{a_{i}}\left(y_{j}\right)=\operatorname{Lim}_{i} \operatorname{Lim}_{j} C_{\{0\}}\left(y_{j} a_{i}\right)=0 .
$$

Then there exists $N$ such that for each $i \geq N$ there exists $M(i)$ such that, for $j \geq M(i), C_{\{0\}}\left(y_{j} a_{i}\right)=0$ for $j \geq M(i)$. This means $y_{j} a_{i} \neq 0$, so $y_{j}=a_{i}$, for $j \geq M(i)$. Taking $i=N$ and $M=M(N)$ we have

$$
Y_{M}=Y_{M+1}=\cdots=a_{N} .
$$

But then on taking $i=N+P$ and applying the above we have $a_{N+P}=a_{N}$. So $a_{N}=a_{N+1}=\cdots$. But this implies that

$$
\operatorname{Lim}_{j} \operatorname{Lim}_{i} C_{\{0\}}^{a_{i}}\left(y_{j}\right)=\operatorname{Lim}_{j} \operatorname{Lim}_{i} C_{\{0\}}\left(y_{j} a_{i}\right)=0 \text {. }
$$

A similar argument gives that if $\operatorname{Lim}_{j} \operatorname{Lim}_{i} C_{\{0\}}^{a_{i}}\left(y_{j}\right)=0$ then $\operatorname{Lim}_{i} \operatorname{Lim}_{j} C_{\{0\}}^{a_{i}}\left(y_{j}\right)$ exists and is also 0 . We have in this subcase, $t=0$, that if one of the iterated limits exists and is 0 then the other must exist and be equal to 0 .

Suppose now that one of the iterated limits exists and is 1 . Then if the other iterated limit exists, it too must be 1 , since if it were 0 , the first iterated limit would also have to equal 0 , by the result of the previous paragraph.

In summary then we have that, for $t=0$, if the iterated limits both exist, they must be equal.

Suppose B: Suppose $\left\{a_{i}\right\}$ has an infinite number of 0 's or 1's.

I. Consider $C_{\{1\}}^{a_{i}}\left(y_{j}\right)=C_{\{1\}}\left(y_{j} a_{i}\right)$. Note that $\operatorname{Lim}_{i} \operatorname{Lim}_{j} C_{\{1\}}\left(y_{j} a_{i}\right)=1$ is impossible, and $\operatorname{Lim}_{j} \operatorname{Lim}_{i} C_{\{1\}}\left(y_{j} a_{i}\right)=1$ is impossible. So if both of the iterated limits exist, they both must be 0 and hence equal. A similar argument of course applies if $\left\{y_{j}\right\}$ has an infinite number of 0 's or 1's.

This together with Case A gives, by Grothendieck's Theorem, that $C_{\{1\}}$ is weakly almost periodic.

II. Consider $C_{\{0\}}^{a_{i}}\left(y_{j}\right)=C_{\{0\}}\left(y_{j} a_{i}\right)$. Note that $\operatorname{Lim}_{j} \operatorname{Lim}_{i} C_{\{0\}}\left(y_{j} a_{i}\right)=0$ and $\operatorname{Lim}_{i} \operatorname{Lim}_{j} C_{\{0\}}\left(y_{j} a_{i}\right)=0$ are impossible. So if both of the iterated limits exist, they must be 1 and therefore equal. So by Case $A$ and Grothendieck's Theorem $C_{\{0\}}$ is weakly almost periodic. Suppose $A \subset S$ and $x \in S$. Then $C_{A}^{x}=C_{A x-1}$ 
where $A x^{-1}=\{t \mid t x \in A\}$. So we see that if $A$ does not contain 0 or 1 then $A x^{-1}=\varnothing$ for all $x \in S$. Therefore $\left\{C_{A}^{x} \mid x \in S\right\}=\left\{C_{\phi}\right\}$. Therefore $C_{A}$ is weakly almost periodic.

If $0 \in A$ and $1 \notin A$ then $A x^{-1}=\{0\} x^{-1}$.

If $1 \in A$ and $0 \notin A$ then $A x^{-1}=\{1\} x^{-1}$.

If $0,1 \in A$ then $A x^{-1}=S$ so then $\left\{C_{A}^{x} \mid x \in S\right\}=\left\{C_{S}\right\}$ and $C_{A}$ is weakly almost periodic.

Therefore in checking to see if $C_{A}$ is weakly almost periodic we need only consider the subsets $A=\{0\}$ and $A=\{1\}$. So then since $C_{\{0\}}$ and $\dot{C}_{\{1\}}$ are weakly almost periodic we have $m(S)=W(S)$ (Theorem 2).

We have already noted that $m(S) \neq A(S)$ and so the theorem is proved.

Corollary. Let $S$ be a semigroup such that $\odot$ is separately continuous on $\beta(S)$. Then $\odot$ need not be jointly continuous.

Proof. Let $S$ be as in Theorem 4 with the discrete topology. Since $m(S)=$ $W(S)$ and $m(S) \neq A(S)$, Theorem 6.2 of Pym [8] and the remark in $\$ 2$ give that $\odot$ is separately but not jointly continuous.

We note that an open problem has been the question: Let $S$ be a semigroup. Are the following two properties on $S$ equivalent?

(a) $S$ has c.s.p.

(b) Convolution is identical with evolution on $m(S)^{*}$.

By Theorem 2 of $\$ 2$ we have (b) is equivalent to:

(c) $S$ has c.s.s.p.

So (a) implies (b) since trivially (a) implies (c). But Corollary 1 above serves as a counterexample to "(b) implies (a)," thus resolving the question in the negative.

\section{BIBLIOGRAPHY}

1. R. F. Arens, The adjoint of a bilinear operation, Proc. Amer. Math. Soc. 2 (1951), 839-848. MR 13, 659.

2. K. deLeeuw and I. Glicksberg, Applications of almost periodic compactifications, Acta. Math. 105 (1961), 63-97. MR 24 \# A1632.

3. A. Grothendieck, Critères de compacité dans les espaces fonctionnels généraux, Amer. J. Math. 74 (1952), 168-186. MR 13, 857.

MR 16, 1136.

4. John L. Kelley, General topology, Van Nostrand, Princeton, N. J., 1955.

5. Lynn H. Loomis, An introduction to abstract harmonic analysis, Van Nostrand, Princeton, N. J., 1953. MR 14, 883.

6. H. Mankowitz, Unpublished manuscript (untitled).

7. John S. Pym, The convolution of linear functionals, Proc. London Math. Soc. (3) 14 (1964), 431-444. MR 31 \#617.

8. - The convolution of functionals on spaces of bounded functions, Proc. London Math. Soc. (3) 15 (1965), 84-104. MR 30 \#3367.

DEPARTMENT OF MATHEMATICS, TEMPLE UNIVERSITY, PHILADELPHIA, PENNSYLVANIA 